\title{
EMBEDDING FUNCTIONS AND THEIR ROLE IN INTERPOLATION THEORY
}

\author{
EVGENIY PUSTYLNIK
}

\begin{abstract}
The embedding functions of an intermediate space $A$ into a Banach couple $\left(A_{0}, A_{1}\right)$ are defined as its embedding constants into the couples $\left(\frac{1}{\alpha} A_{0}, \frac{1}{\beta} A_{1}\right), \forall \alpha, \beta>0$. Using these functions, we study properties and interrelations of different intermediate spaces, give a new description of all real interpolation spaces, and generalize the concept of weak-type interpolation to any Banach couple to obtain new interpolation theorems.
\end{abstract}

\section{INTRODUCTION}

The interpolation theory arose from a general problem of studying linear operators on large collections of Banach spaces. First those are spaces with a good analytical description (expression of norm) depending on a numerical parameter, such as $L_{p}, \operatorname{Lip} \alpha, W_{p}^{k}$ etc. [11]. As a natural generalization, one then took families of spaces with a function parameter [17] or some other common characteristics — for example, symmetric (rearrangement invariant) spaces [21]. Impetuous development of the interpolation theory generated soon a problem of this theory fundamentals and basic notions (see e.g. a classical work [1]). So one get the totality of all intermediate spaces for a Banach couple as a basic and largest object, from which one may extract spaces with different interpolation properties.

A Banach space $A$ is called intermediate for a Banach couple $\overrightarrow{\mathbf{A}}=\left(A_{0}, A_{1}\right)$ if $\Delta(\overrightarrow{\mathbf{A}}) \subset A \subset \Sigma(\overrightarrow{\mathbf{A}})$, where $\Delta(\overrightarrow{\mathbf{A}})=A_{0} \cap A_{1}, \Sigma(\overrightarrow{\mathbf{A}})=A_{0}+A_{1}$ with the standard definition of norms. The totality of all such spaces will be denoted by $\pi(\overrightarrow{\mathbf{A}})$. The indicated embeddings are always accompanied by the norm inequalities

(1) $\|x\|_{A} \geq D\|x\|_{\Sigma(\overrightarrow{\mathbf{A}})} \quad(\forall x \in A), \quad\|x\|_{A} \leq C\|x\|_{\Delta(\overrightarrow{\mathbf{A}})} \quad(\forall x \in \Delta(\overrightarrow{\mathbf{A}}))$.

1991 Mathematics Subject Classification. Primary: 46B70.

Key words and phrases. Embedding, interpolation, $K$-space, operator of weak type.

This research was supported by the Center of Scientific Absorption of the Ministry of Absorption of the State of Israel.

Received: April 15, 1996

(C) 1996 Mancorp Publishing, Inc. 
A combination $\left(A_{0}, A_{1}, A\right)=(\overrightarrow{\mathbf{A}}, A)$ with $A \in \pi(\overrightarrow{\mathbf{A}})$ will be called a triple. If $\overrightarrow{\mathbf{A}}, \overrightarrow{\mathbf{B}}$ are two Banach couples, then the notation $T: \overrightarrow{\mathbf{A}} \rightarrow \overrightarrow{\mathbf{B}}$ means that a linear operator $T$ acts from $\Sigma(\overrightarrow{\mathbf{A}})$ into $\Sigma(\overrightarrow{\mathbf{B}})$ such that

$$
\|T\|_{A_{i} B_{i}}=\sup _{x \in A_{i}}\|T x\|_{B_{i}} /\|x\|_{A_{i}}<\infty, \quad i=0,1 .
$$

If for any such operator and for some spaces $A \in \pi(\overrightarrow{\mathbf{A}}), B \in \pi(\overrightarrow{\mathbf{B}})$, the inequality

$$
\|T\|_{A B} \leq M \max _{i=0,1}\|T\|_{A_{i} B_{i}}
$$

holds, we say the triple $(\overrightarrow{\mathbf{A}} A)$ is interpolation with respect to the triple $(\overrightarrow{\mathbf{B}}, B)$ (in the case $M=1$ we shall speak about exact interpolation). In the case $\overrightarrow{\mathbf{A}}=\overrightarrow{\mathbf{B}}, A=B$ the space $A$ is said to be interpolation for the Banach couple $\overrightarrow{\mathbf{A}}$, and the totality of all such spaces will be denoted by $\operatorname{Int}(\overrightarrow{\mathbf{A}})$.

For an element $y \in \Delta(\overrightarrow{\mathbf{B}})$ and a functional $f \in(\Sigma(\overrightarrow{\mathbf{A}}))^{*}$, we define an one-dimensional operator $T x=f(x) y$ which is obviously bounded from $\overrightarrow{\mathbf{A}}$ to $\overrightarrow{\mathbf{B}}$. We say the triple $(\overrightarrow{\mathbf{A}}, A)$ is partly interpolation with respect to the triple $(\overrightarrow{\mathbf{B}}, B)$, if the inequality (2) is fulfilled for all one-dimensional operators as above. If $(\overrightarrow{\mathbf{A}}, A)=(\overrightarrow{\mathbf{B}}, B)$, then the space $A$ will be called partly interpolation, and the totality of all such spaces will be denoted by $\operatorname{Part}(\overrightarrow{\mathbf{A}})$. We have the following chain of embeddings: $\operatorname{Int}(\overrightarrow{\mathbf{A}}) \subset \operatorname{Part}(\overrightarrow{\mathbf{A}}) \subset$ $\pi(\overrightarrow{\mathbf{A}})$, and besides no two totalities coincide here, unless a couple $\overrightarrow{\mathbf{A}}$ is trivial (i.e. $\Delta(\overrightarrow{\mathbf{A}})$ is closed in both $A_{0}$ and $A_{1}$ ).

Plenty of literature is devoted to the study of interpolation spaces and triples [14]. As a rule, the papers describe different interpolation constructions, and it would be a good luck if a particular space of a practical problem would correspond to one of such descriptions. Otherwise, one need some methods to estimate at least the proximity of a given space to interpolation one. Estimation of the position of an intermediate space relatively to other spaces was studied in [19] (see also references given there) by the use of so-called fundamental functions

$$
\varphi(t, A, \overrightarrow{\mathbf{A}})=\sup _{\substack{\|x\|_{A_{0}} \leq 1 \\\|x\|_{A_{1}} \leq t}}\|x\|_{A}, \psi(t, A, \overrightarrow{\mathbf{A}})=\varphi\left(t, A^{*}, \overrightarrow{\mathbf{A}}^{*}\right), \overrightarrow{\mathbf{A}}^{*}=\left(A_{1}^{*}, A_{0}^{*}\right)
$$

proposed yet in [7]. Another possibility was also mentioned in Introduction to [19] — to use the most natural characteristics of an intermediate space such as its embedding constants into the couple $\overrightarrow{\mathbf{A}}$, i.e. the optimal numbers $C, D$ in the inequalities (1). Such an approach proves to be especially powerful if, together with a given couple $\overrightarrow{\mathbf{A}}$, to consider all couples $\left(\alpha A_{0}, \beta A_{1}\right)$ with any positive numbers $\alpha, \beta$. The space $A$ remains to be intermediate 
for all such couples, while the embedding constants turn into functions of $\alpha, \beta$. The information contained in these functions suffices to get various conclusions on interpolation and other close properties of the space $A$.

An idea to pass from a single couple $\left(A_{0}, A_{1}\right)$ to collection of couples $\left(\alpha A_{0}, \beta A_{1}\right)$ is due to J. Peetre [16], who introduced and systematically used the functionals

$$
J(\alpha, \beta, x, \overrightarrow{\mathbf{A}})=\|x\|_{\alpha A_{0} \cap \beta A_{1}}, \quad K(\alpha, \beta, x, \overrightarrow{\mathbf{A}})=\|x\|_{\alpha A_{0}+\beta A_{1}}
$$

(in fact, J.Peetre considered only couples $\left(A_{0}, t A_{1}\right)$; the simultaneous usage of two parameters is due to $\mathrm{V}$. Ovchinnikov [15]). As functions of $\alpha, \beta$ these functionals belong to the class of continuous nondecreasing functions homogeneous of degree 1; this class will be denoted below by $\mathfrak{M}$.

The present paper develops the above mentioned idea from Introduction to [19]. It consists of four sections. In Section 1 we define and study the notion of embedding functions $C(\alpha, \beta)$ and $D(\alpha, \beta)$ (including some examples). In particular, we obtain the necessary and sufficient conditions for the inequality $C(\alpha, \beta) \leq D(\alpha, \beta)$. A similar result was obtained by $\mathrm{A}$. Dmitriev [7] for the fundamental functions (3) but only for the case of regular couple $\overrightarrow{\mathbf{A}}$, i.e. when $\Delta(\overrightarrow{\mathbf{A}})$ is dense in $A_{0}$ and $A_{1}$. Our proof does not use the conjugate couple $\overrightarrow{\mathbf{A}}^{*}$ and need not such density. In the next section we study the sum and the intersection of arbitrary set of intermediate spaces and show that, together with the multiplication by number, these operations allow, starting from the initial spaces of the couple $A_{0}, A_{1}$, to obtain all spaces of the real interpolation. As a tool for doing this we use the maximal and the minimal intermediate spaces with given embedding functions.

Sections 3 and 4 are devoted to the weak-type interpolation in arbitrary Banach couples which is defined here as follows: we say a linear operator $T: A \rightarrow B$ is of weak type $(\Phi, \Psi)$, if all we know about these spaces is that $C_{A}(\alpha, \beta) \leq \Phi(\alpha, \beta), D_{B}(\alpha, \beta) \geq \Psi(\alpha, \beta)$. The weak-type interpolation relates to an operator being simultaneously of two weak types $\left(\Phi_{0}, \Psi_{0}\right)$ and $\left(\Phi_{1}, \Psi_{1}\right)$. In Section 3 we describe the spaces which can be obtained from this fact by a functorial approach, while the given in Section 4 generalization of the Krein-Semenov theorem [13] may be regarded as the main result of the paper. It is stronger than the former one even in the case of the couple $\left(L_{1}, L_{\infty}\right)$ considered by the authors of this method.

\section{EMBEDDING FUNCTIONS AND THEIR PROPERTIES}

Now we consider the embedding constants of an intermediate space $A$ into all couples $\left(\alpha A_{0}, \beta A_{1}\right), \alpha, \beta>0$. For many purposes, it will be more convenient to take the couples in the form $\left(\frac{1}{\alpha} A_{0}, \frac{1}{\beta} A_{1}\right)$ and define the embedding constants as

$$
C(\alpha, \beta)=\sup _{x \in \Delta(\overrightarrow{\mathbf{A}})} \frac{\|x\|_{A}}{\|x\|_{\frac{1}{\alpha} A_{0} \cap \frac{1}{\beta} A_{1}}}, \quad D(\alpha, \beta)=\inf _{x \in A} \frac{\|x\|_{A}}{\|x\|_{\frac{1}{\alpha} A_{0}+\frac{1}{\beta} A_{1}}} .
$$


By such a choice we have obtained two functions from $\mathfrak{M}$ which will be called embedding functions of a space $A$ into the couple $\overrightarrow{\mathbf{A}}$. At first sight they seems to be not having any connection with each other. As we shall see further, this is not true even in general case. And in special cases the connection between them can be very close. Let us see these functions in some examples.

Example 1. Let $E$ be a rearrangement invariant space (see e.g. [12]) with the fundamental function $\varphi(t)$ which is defined as $\left\|\chi_{G}\right\|_{E}$ with mes $G=t$ (for instance, $L_{p}$ has a fundamental function $t^{1 / p}$ ). Consider this space as intermediate one for the couple $\overrightarrow{\mathbf{L}}=\left(L_{1}, L_{\infty}\right)$. Then after not difficult but tedious computation, we obtain that

$$
C(\alpha, \beta)=D(\alpha, \beta)=\beta \varphi(\alpha / \beta) .
$$

Example 2. Take a couple of weight spaces $\left(E^{v}, E^{w}\right)$ with continuous positive weights $v(t), w(t)$ on $(0, \infty)$. If for some constants $k_{1}, k_{2}>0$

$$
k_{1} \min (v(t), w(t)) \leq z(t) \leq k_{2} \max (v(t), w(t)), \quad \forall t>0,
$$

then the space $E^{z}$ is intermediate for this couple and

$$
\begin{aligned}
& C(\alpha, \beta) \sim \sup _{t} z(t) \min (\alpha / v(t), \beta / w(t)), \\
& D(\alpha, \beta) \sim \inf _{t} z(t) \max (\alpha / v(t), \beta / w(t))
\end{aligned}
$$

(equivalence with constant 2). If $z=\Phi(v, w)$ with some $\Phi \in \mathfrak{M}$, then

$$
C(\alpha, \beta) \sim D(\alpha, \beta) \sim \Phi(\alpha, \beta) .
$$

Sometimes it is enough only to estimate $C$ and $D$ functions from one side. If we can find out that for some $\Phi \in \mathfrak{M}$ and for any $x \in \Delta(\overrightarrow{\mathbf{A}})$

$$
\|x\|_{A} \leq \Phi\left(\|x\|_{A_{0}},\|x\|_{A_{1}}\right),
$$

then $C(\alpha, \beta) \leq \Phi(\alpha, \beta)$. For estimating $D$-function, one may use the duality between sums and intersections. If the couple $\overrightarrow{\mathbf{A}}$ is regular and $\Delta(\overrightarrow{\mathbf{A}})$ is dense in $A$, we can pass to the conjugate spaces. If now for some $\Psi \in \mathfrak{M}$ and for any $f \in \Delta\left(\overrightarrow{\mathbf{A}}^{*}\right)=(\Sigma(\overrightarrow{\mathbf{A}}))^{*}$ we have an inequality

$$
\|f\|_{A^{*}} \leq \Psi\left(\|f\|_{A_{0}^{*}},\|f\|_{A_{1}^{*}}\right),
$$

then $D(\alpha, \beta) \geq 1 / \Psi(1 / \alpha, 1 / \beta)$.

To prove this assertion we fix $x \in \Sigma(\overrightarrow{\mathbf{A}})$ and take $f \in(\Sigma(\overrightarrow{\mathbf{A}}))^{*}$ such that

$$
f(x)=\|x\|_{\frac{1}{\alpha} A_{0}+\frac{1}{\beta} A_{1}}, \quad\|f\|_{\alpha A_{0}^{*} \cap \beta A_{1}^{*}}=1 .
$$


This is possible because $\left(\frac{1}{\alpha} A_{0}+\frac{1}{\beta} A_{1}\right)^{*}=\alpha A_{0}^{*} \cap \beta A_{1}^{*}$. Then $\|f\|_{A_{0}^{*}} \leq$ $1 / \alpha,\|f\|_{A_{1}^{*}} \leq 1 / \beta$. So, for any $x \in A$ we obtain

$$
\begin{aligned}
\frac{\|x\|_{A}}{\|x\|_{\frac{1}{\alpha} A_{0}+\frac{1}{\beta} A_{1}}}=\frac{\|x\|_{A}}{|f(x)|} & \geq \inf _{x \in A} \frac{\|x\|_{A}}{|f(x)|}=\frac{1}{\|f\|_{A^{*}}} \\
& \geq \frac{1}{\Psi\left(\|f\|_{A_{0}^{*}},\|f\|_{A_{1}^{*}}\right)} \geq \frac{1}{\Psi(1 / \alpha, 1 / \beta)},
\end{aligned}
$$

that proves the assertion after infimizing over all $x \in A$.

Example 3. Let $\mathrm{T}$ be an unbounded selfadjoint operator in a Hilbert space $H$ such that $(T x, x)>0$ for all non-zero $x \in H$. Consider couple $A_{0}=$ $H, A_{1}=\mathcal{D}(T)$ with the norm $\|x\|_{A_{1}}=\|T x\|$. For any increasing concave function $\varphi(t)$, we can define an operator function $\varphi(T)$ with the domain $\mathcal{D}(\varphi(T))$, which we regard as a Banach space $A_{\varphi}$ with the norm $\|x\|_{A_{\varphi}}=$ $\|\varphi(T) x\|$. Using the integral representation of $\varphi(T)$ and Jensen inequality, we obtain the so-called "moment inequality"

$$
\|\varphi(T) x\| \leq\|x\| \varphi(\|T x\| /\|x\|),
$$

which means that

$$
\|x\|_{A_{\varphi}} \leq\|x\|_{A_{0}} \varphi\left(\|x\|_{A_{1}} /\|x\|_{A_{0}}\right) .
$$

This gives us immediately that $C(\alpha, \beta) \leq \alpha \varphi(\beta / \alpha)$.

In order to estimate $D(\alpha, \beta)$ we pass to the dual couple $\left(A_{0}^{*}, A_{1}^{*}\right)$ with $A_{0}^{*}=H$ and $A_{1}^{*}=\mathcal{D}\left(T^{-1}\right.$ ) (or the completion of $H$ with respect to the norm $\left\|T^{-1} x\right\|$ if $T^{-1}$ is a bounded operator). By the analogous way we define the space $A_{\varphi}^{*}$ and obtain that

$$
\left\|\varphi^{-1}(T) x\right\| \leq \frac{\|x\|}{\varphi\left(\|x\| /\left\|T^{-1} x\right\|\right)}
$$

or in other words

$$
\|x\|_{A_{\varphi}^{*}} \leq \frac{\|x\|_{A_{0}^{*}}}{\varphi\left(\|x\|_{A_{0}^{*}} /\|x\|_{A_{1}^{*}}\right)} .
$$

This gives us that $D(\alpha, \beta) \geq \alpha \varphi(\beta / \alpha)$, hence $C(\alpha, \beta) \leq D(\alpha, \beta)$.

The last inequality is not occasional. It is valid for any interpolation space and even for a larger class of spaces, that will be seen from the next theorem.

Theorem 1. In order that $C(\alpha, \beta) \leq D(\alpha, \beta)$ for all $\alpha, \beta>0$ it is necessary and sufficient that $A$ be an exact partly interpolation space for the couple $\overrightarrow{\mathbf{A}}$. Proof. a)sufficiency. We have to prove that, for any two elements $x \in A$ and $y \in A_{o} \cap A_{1}$, we always have

$$
\frac{\|y\|_{A}}{\|y\|_{\frac{1}{\alpha} A_{0} \cap \frac{1}{\beta} A_{1}}} \leq \frac{\|x\|_{A}}{\|x\|_{\frac{1}{\alpha} A_{0}+\frac{1}{\beta} A_{1}}} .
$$


Without loss of generality, we may take $\|y\|_{\frac{1}{\alpha} A_{0} \cap \frac{1}{\beta} A_{1}}=1$. Now let us take a functional $f(x)$ with norm 1 in the conjugate space $\left(\frac{1}{\alpha} A_{0}+\frac{1}{\beta} A_{1}\right)$ * and such that $f(x)=\|x\|_{\frac{1}{\alpha} A_{0}+\frac{1}{\beta} A_{1}}$. Consider the one-dimensional operator $T z=f(z) y$ and show that the restrictions of this operator on the spaces $A_{0}, A_{1}$ have the norms not greater than 1 . Indeed,

$$
\|T z\|_{A_{0}}=|f(z)|\|y\|_{A_{0}} \leq \alpha\|z\|_{\frac{1}{\alpha} A_{0}+\frac{1}{\beta} A_{1}} \leq\|z\|_{A_{0}}
$$

and so for $A_{1}$. Thus $\|T x\|_{A} \leq\|x\|_{A}$ and from this

$$
\|y\|_{A} \leq \frac{\|x\|_{A}}{|f(x)|}=\frac{\|x\|_{A}}{\|x\|_{\frac{1}{\alpha} A_{0}+\frac{1}{\beta} A_{1}}}
$$

which proves (4).

b)necessity. Let (4) is true for any $x \in A, y \in A_{0} \cap A_{1}$. Take some one-dimensional operator $T x=f(x) y$ and define

$$
M_{i}=\sup _{x \in A_{i}} \frac{|f(x)|}{\|x\|_{A_{i}}}, \quad i=0,1 .
$$

Then the norm of $T$ on the space $A_{i}$ is equal to $M_{i}\|y\|_{A_{i}}$. We have to show that

$$
\|T x\|_{A} \leq \max _{i=0,1} M_{i}\|y\|_{A_{i}}\|x\|_{A} .
$$

It follows from (4) that

$$
\|T x\|_{A}=|f(x)|\|y\|_{A} \leq \frac{|f(x)|}{\|x\|_{\frac{1}{\alpha} A_{0}+\frac{1}{\beta} A_{1}}}\|y\|_{\frac{1}{\alpha} A_{0} \cap \frac{1}{\beta} A_{1}}\|x\|_{A} .
$$

Now we show that

$$
\frac{|f(x)|}{\|x\|_{\frac{1}{\alpha} A_{0}+\frac{1}{\beta} A_{1}}} \leq \max \left(\alpha M_{0}, \beta M_{1}\right) .
$$

Indeed, take arbitrary $\varepsilon>0$ and let $x_{0} \in A_{0}, x_{1} \in A_{1}$ be such that $x=$ $x_{0}+x_{1}$ and

$$
\|x\|_{\frac{1}{\alpha} A_{0}+\frac{1}{\beta} A_{1}} \geq(1-\varepsilon)\left(\left\|x_{0}\right\|_{\frac{1}{\alpha} A_{0}}+\left\|x_{1}\right\|_{\frac{1}{\beta} A_{1}}\right) .
$$

Then

$$
\begin{aligned}
\frac{|f(x)|}{\|x\|_{\frac{1}{\alpha} A_{0}+\frac{1}{\beta} A_{1}}} & \leq \frac{\left|f\left(x_{0}\right)\right|+\left|f\left(x_{1}\right)\right|}{(1-\varepsilon)\left(\frac{1}{\alpha}\left\|x_{0}\right\|_{A_{0}}+\frac{1}{\beta}\left\|x_{1}\right\|_{A_{1}}\right)} \\
& \leq \frac{1}{1-\varepsilon} \max \left[\frac{\alpha\left|f\left(x_{0}\right)\right|}{\left\|x_{0}\right\|_{A_{0}}}, \frac{\beta\left|f\left(x_{1}\right)\right|}{\left\|x_{1}\right\|_{A_{1}}}\right] \\
& \leq \frac{1}{1-\varepsilon} \max \left(\alpha M_{0}, \beta M_{1}\right),
\end{aligned}
$$


and it remains only to pass to limit as $\varepsilon \rightarrow 0$.

So, we have

$$
\|T x\|_{A} \leq \max \left(\alpha M_{0}, \beta M_{1}\right) \max \left(\frac{1}{\alpha}\|y\|_{A_{0}}, \frac{1}{\beta}\|y\|_{A_{1}}\right)\|x\|_{A} .
$$

Now we put $\alpha=M_{1}, \beta=M_{0}$. Then

$$
\begin{aligned}
\|T x\|_{A} & \leq M_{0} M_{1} \max \left[\frac{\|y\|_{A_{0}}}{M_{1}}, \frac{\|y\|_{A_{1}}}{M_{0}}\right]\|x\|_{A} \\
& =\max \left(M_{0}\|y\|_{A_{0}}, M_{1}\|y\|_{A_{1}}\right)\|x\|_{A} \cdot \mathbf{\cdot}
\end{aligned}
$$

If the interpolation constant of the space $A$ is greater than 1 , the inequality for $C$ and $D$ function is fulfilled up to equivalence. The inverse inequality happens more seldom; it is caused not by properties of a single space but by those of the couple on the whole. We give only one result with sufficient condition.

Theorem 2. If a couple $\overrightarrow{\mathbf{A}}$ satisfies inequality

$$
\inf _{\alpha, \beta} \sup _{x \in \Delta(\overrightarrow{\mathbf{A}})} \frac{\|x\|_{\alpha A_{0}+\beta A_{1}}}{\|x\|_{\alpha A_{0} \cap \beta A_{1}}} \geq k>0
$$

then for any intermediate space $D(\alpha, \beta) \leq \frac{1}{k} C(\alpha, \beta)$.

Proof. Let $\alpha, \beta>0$ be fixed. For arbitrary $\varepsilon>0$ we can find $x \in \Delta(\overrightarrow{\mathbf{A}})$ such that $\|x\|_{\alpha A_{0}+\beta A_{1}} \geq(k-\varepsilon)\|x\|_{\alpha A_{0} \cap \beta A_{1}}$. It follows then immediately from the definitions of $C$ and $D$ functions that

$$
\frac{D(1 / \alpha, 1 / \beta)}{C(1 / \alpha, 1 / \beta)} \leq \frac{\|x\|_{\alpha A_{0} \cap \beta A_{1}}}{\|x\|_{\alpha A_{0}+\beta A_{1}}} \leq \frac{1}{k-\varepsilon},
$$

and this proves our theorem if $\varepsilon$ tends to 0 .

If in Theorem 2 we have $k=1$, then for any exact interpolation (and even partly interpolation space), we obtain an equality $D(\alpha, \beta)=C(\alpha, \beta)$. The couple $\left(L_{1}, L_{\infty}\right)$ from Example 1 is quite so. As another example we can take the couple $\left(C, C^{1}\right)$ on $[0,1]$.

Further on we shall index the embedding functions when studying different intermediate spaces in the same Banach couple. It is evident that an exact embedding $A \subset B$ entails inequalities $C_{A}(\alpha, \beta) \geq C_{B}(\alpha, \beta)$ and $D_{A}(\alpha, \beta) \geq D_{B}(\alpha, \beta)$. The inverse implication is not true, and even the way for obtaining inverse relations is not clear. Nevertheless, such relations do exist, and the following theorem is valid. 
Theorem 3. Let $A, B \in \pi(\overrightarrow{\mathbf{A}})$ and

$$
\int_{0}^{\infty} \frac{C_{B}(\alpha, \beta)}{D_{A}(\alpha, \beta)} \frac{d \beta}{\beta}=\int_{0}^{\infty} \frac{C_{B}(\alpha, \beta)}{D_{A}(\alpha, \beta)} \frac{d \alpha}{\alpha}<\infty .
$$

Then $A \subset B$. In the case of exact embedding $A_{0} \subset A_{1}$ the limits of the first integral are from 0 to $\alpha$, of the second from $\beta$ to $\infty$. If $A_{1} \subset A_{0}$ (exactly) then the limits of the first integral are from $\alpha$ to $\infty$, of the second from 0 to $\beta$.

Notice that the equality of integrals is not an additional condition. It always follows from the fact that all $C$ and $D$ functions are homogeneous of degree one.

Proof. Actually, this theorem was proved in [18] in the terms of fundamental functions (3) satisfying inequality

$$
\int_{0}^{\infty} \frac{\varphi(t, B) \psi(t, A)}{t^{2}} d t<\infty .
$$

In order to obtain the present wording it is enough to use the connection between embedding and fundamental functions

$$
\varphi(t, B)=C_{B}(1, t), \quad \psi(t, A)=t / D_{A}(1, t)
$$

and the homogeneity of functions $C(\alpha, \beta)$ and $D(\alpha, \beta)$.

Let us say a couple $\overrightarrow{\mathbf{A}}$ is $C$-abundant, if for any $\Phi(\alpha, \beta) \in \mathfrak{M}$ there exists a space $A \in \pi(\overrightarrow{\mathbf{A}})$ with $C_{A}(\alpha, \beta) \sim \Phi(\alpha, \beta)$. The $D$-abundance is defined by the same way. The couples $\left(L_{1}, L_{\infty}\right)$ from Example 1 and $\left(E^{v}, E^{w}\right)$ from Example 2 have abundance of the both types. Some criteria of abundance (in the terms of fundamental functions) are given in [19]. For any type of abundance (and even under some weaker conditions), the embedding of $A$ into $B$ is proper, i.e. $A \neq B$. This means that in such a couple there is no space $A$, for which the ratio $C(1, t) / D(1, t)$ is integrable with respect to the measure $d t / t$.

\section{Sum AND Intersection OF BANACH SPACES}

Embedding functions give a natural way to define and to construct the maximal and the minimal intermediate spaces from [18] (last time they usually are called (generalized) Lorentz and Marcinkiewicz spaces respectively). Roughly speaking, for an arbitrary function $\Phi(\alpha, \beta) \in \mathfrak{M}$, a maximal (Lorentz) space $\Lambda_{\Phi}(\overrightarrow{\mathbf{A}})$ is the "narrowest" one for which $C(\alpha, \beta) \leq \Phi(\alpha, \beta)$, and a minimal (Marcinkiewicz) space $\mathrm{M}_{\Phi}(\overrightarrow{\mathbf{A}})$ is the "widest" one for which $D(\alpha, \beta) \geq \Phi(\alpha, \beta)$. More exact description of these spaces and of their role in the interpolation theory may be given via generalized sums and intersections of Banach spaces. 
The usual operations of sum and intersection of two spaces together with the multiplication by number are the simplest operations preserving the interpolation property of spaces (and even the interpolation constant). In particular, all above considered spaces $\alpha A_{0} \cap \beta A_{1}$ and $\alpha A_{0}+\beta A_{1}$ are exact interpolation for the couple $\overrightarrow{\mathbf{A}}$. Following [1], these operations may be generalized to the case of arbitrary many spaces.

The intersection $\Delta\left(A_{\omega}\right), \omega \in \Omega$, is the set of elements $x$ belonging to any $A_{\omega}$ with $\sup _{\omega}\|x\|_{A_{\omega}}<\infty$, and this supremum is taken as the norm in $\Delta\left(A_{\omega}\right)$. The sum $\Sigma\left(A_{\omega}\right)$ is defined as the intersection of all Banach spaces in which any $A_{\omega}$ is embedded exactly, provided that at least one of such spaces really exists. Thus we have that

$$
\|x\|_{\Sigma\left(A_{\omega}\right)} \leq \inf _{\omega}\|x\|_{A_{\omega}}
$$

for any $x$ common for all $A_{\omega}$. For a given set of spaces $\left\{A_{\omega}\right\}$ to be summable, it is sufficient that they are intermediate for some couple $\overrightarrow{\mathbf{A}}$, and their embedding constants into the space $\Sigma(\overrightarrow{\mathbf{A}})$ are bounded on the whole (we say they are uniformly embedded into $\Sigma(\overrightarrow{\mathbf{A}})$ ).

It is also possible to give a direct construction of the sum as a collection of all $x$ representable in a form $x=\sum x_{\omega}$, where $x_{\omega} \in A_{\omega}, \omega \in \Omega$ and $\sum\left\|x_{\omega}\right\|_{A_{\omega}}<\infty$ (it follows from this immediately that the set of $x_{\omega} \neq 0$ is at most countable). The norm in a space thus obtained is defined as $\inf \sum\left\|x_{\omega}\right\|_{A_{\omega}}$ over all possible representation of $x$ as above.

If all $A_{\omega} \in \pi(\overrightarrow{\mathbf{A}})$ then both $\Delta\left(A_{\omega}\right)$ and $\Sigma\left(A_{\omega}\right)$ also are intermediate for the couple $\overrightarrow{\mathbf{A}}$. We have even a more general assertion.

Theorem 4. Let each triple $\left(\overrightarrow{\mathbf{A}}, A_{\omega}\right), \omega \in \Omega$, be exact interpolation for the corresponding triple $\left(\overrightarrow{\mathbf{B}}, B_{\omega}\right)$. Then the triple $\left(\overrightarrow{\mathbf{A}}, \Delta\left(A_{\omega}\right)\right)$ is exact interpolation for the triple $\left(\overrightarrow{\mathbf{B}}, \Delta\left(B_{\omega}\right)\right)$, and the triple $\left(\overrightarrow{\mathbf{A}}, \Sigma\left(A_{\omega}\right)\right)$ is exact interpolation for the triple $\left(\overrightarrow{\mathbf{B}}, \Sigma\left(B_{\omega}\right)\right)$.

Proof. If we write the inequality $\|T x\|_{B_{\omega}} \leq\|x\|_{A_{\omega}}$ for all $\omega \in \Omega$ and take supremum of both sides, we immediately obtain the first assertion of the theorem. To prove the second assertion, we define the space $D \in \pi(\overrightarrow{\mathbf{A}})$ with the norm $\|x\|_{D}=\frac{1}{2}\left(\|x\|_{\Sigma\left(A_{\omega}\right)}+\|T x\|_{\Sigma\left(B_{\omega}\right)}\right)$ and see that each summand in the brackets is not greater than $\|x\|_{A_{\omega}}$ for each $\omega$. Thus any $A_{\omega}$ is exactly embedded in $D$, and so the norm in $D$ must be no greater than the norm in the sum $\Sigma\left(A_{\omega}\right)$. This implies the required inequality $\|T x\|_{\Sigma\left(B_{\omega}\right)} \leq$ $\|x\|_{\Sigma\left(A_{\omega}\right)}$.

The next assertion gives us a partial case of space summing when the parameter is a continuous real variable.

Theorem 5. Let the spaces $A_{t}, a<t<b$, be uniformly embedded in $\Sigma(\overrightarrow{\mathbf{A}})$, and let the space $\Delta(\overrightarrow{\mathbf{A}})$ be dense in any $A_{t}$. Let $\|x\|_{A_{t}}$ be a continuous 
function of $t$ for each $x \in \Delta(\overrightarrow{\mathbf{A}})$. Then the space $\Sigma\left(A_{t}\right)$ consists of all $x \in \Sigma(\overrightarrow{\mathbf{A}})$ representable in the form

$$
x=\int_{a}^{b} u(t) d t
$$

where $u(t)$ is a function with values in $\Delta(\overrightarrow{\mathbf{A}})$ such that the function $\|u(t)\|_{A_{t}}$ is summable with respect to $t$ on $(a, b)$. The norm in $\Sigma(\overrightarrow{\mathbf{A}})$ is given by the equality

$$
\|x\|_{\Sigma\left(A_{t}\right)}=\inf \int_{a}^{b}\|u(t)\|_{A_{t}} d t
$$

where infimum is taken over all representations of $x$ as above.

Notice that $a$ may be equal to zero and $b$ may be equal to infinity.

Proof. Let us denote the space of all $x$ representable in the form (5) with the norm (6) by $A$. Fix some $t=t_{0}$ and consider the functions

$$
u_{\varepsilon}(t)= \begin{cases}x / 2 \varepsilon & \text { if }\left|t-t_{0}\right| \leq \varepsilon \\ 0 & \text { if }\left|t-t_{0}\right|>\varepsilon\end{cases}
$$

where $\varepsilon<\min \left(\left|t_{0}-a\right|,\left|t_{0}-b\right|\right)$. Then (5) is fulfilled for $u=u_{\varepsilon}$, hence

$$
\|x\|_{A} \leq \lim _{\varepsilon \rightarrow 0} \int_{a}^{b}\left\|u_{\varepsilon}(t)\right\| A_{t} d t=\|x\|_{A_{t_{0}}},
$$

in virtue of $\|x\|_{A_{t}}$ being continuous. So any $A_{t}$ is exactly embedded in $A$.

Let now $B$ be some other Banach space such that all $A_{t}$ are exactly embedded in $B$. Then for each $x$ representable in the form (5),

$$
\int_{a}^{b}\|u(t)\|_{A_{t}} d t \geq \int_{a}^{b}\|u(t)\|_{B} d t
$$

thus the integral from (5) converges in $B$ and $x \in B$. Further

$$
\|x\|_{A}=\inf \int_{a}^{b}\|u(t)\|_{A_{t}} d t \geq \inf \left\|\int_{a}^{b} u(t) d t\right\|_{B}=\|x\|_{B},
$$

hence $A$ is exactly embedded in $B$. Since $B$ is arbitrary, this finishes the proof.

Starting from the initial spaces $A_{0}, A_{1}$ and using different combinations of multiplications by number, sums and intersections, one can get a large class of intermediate spaces which we denote by $\operatorname{Prim}(\overrightarrow{\mathbf{A}})$. In virtue of Theorem 4 , all these spaces are exact interpolation for the couple $\overrightarrow{\mathbf{A}}$. Just in this 
class we find the above mentioned spaces $\Lambda_{\Phi}(\overrightarrow{\mathbf{A}}), \mathrm{M}_{\Phi}(\overrightarrow{\mathbf{A}})$, since they may be represented in a form

$$
\begin{aligned}
& \Lambda_{\Phi}(\overrightarrow{\mathbf{A}})=\sum_{\alpha, \beta} \Phi(\alpha, \beta)\left(\frac{1}{\alpha} A_{0} \cap \frac{1}{\beta} A_{1}\right)=\sum_{t} \Phi(1, t)\left(A_{0} \cap \frac{1}{t} A_{1}\right), \\
& \mathrm{M}_{\Phi}(\overrightarrow{\mathbf{A}})={ }_{\alpha, \beta} \Phi(\alpha, \beta)\left(\frac{1}{\alpha} A_{0}+\frac{1}{\beta} A_{1}\right)={ }_{t} \Phi(1, t)\left(A_{0}+\frac{1}{t} A_{1}\right) .
\end{aligned}
$$

By the way, we can get from this representation the formulas for norms in these spaces. Indeed, from the definition of intersection we obtain an expression of the norm in Marcinkiewicz space via $K$-functional of Peetre:

$$
\|x\|_{\mathrm{M}_{\Phi}(\overrightarrow{\mathbf{A}})}=\sup _{t} \Phi(1, t) K\left(1, \frac{1}{t}, x, \overrightarrow{\mathbf{A}}\right) .
$$

Due to Theorem 5, the norm in Lorentz space can be expressed via $J$ functional of Peetre:

$$
\|x\|_{\Lambda_{\Phi}(\overrightarrow{\mathbf{A}})}=\inf \int_{0}^{\infty} \Phi(1, t) J\left(1, \frac{1}{t}, u(t), \overrightarrow{\mathbf{A}}\right) d t,
$$

where infimum is taken over all representation of $x$ in the form

$$
x=\int_{0}^{\infty} u(t) d t, \quad u(t) \in \Delta(\overrightarrow{\mathbf{A}}), \quad \forall t>0 .
$$

For arbitrary $A \in \pi(\overrightarrow{\mathbf{A}})$, the inequality $C_{A}(\alpha, \beta) \leq \Phi(\alpha, \beta)$ is necessary and sufficient for an embedding $A \supset \Lambda_{\Phi}(\overrightarrow{\mathbf{A}})$, and the inequality $D_{A}(\alpha, \beta) \geq$ $\Phi(\alpha, \beta)$ is necessary and sufficient for an embedding $A \subset \mathrm{M}_{\Phi}(\overrightarrow{\mathbf{A}})$.

The spaces from $\operatorname{Prim}(\overrightarrow{\mathbf{A}})$ are closely connected with the real interpolation spaces $(K$-spaces), i.e. spaces having an (equivalent) norm of the type $\|x\|_{A}=\|K(1, t, x, \overrightarrow{\mathbf{A}})\|_{E}$ with some Banach function space (lattice) $E$ defined over $\mathbb{R}^{+}$with the measure $d t / t$ and containing the function $\min (1, t)$ (sometimes $E$ is called a parameter of the real interpolation). The class of such spaces we denote by $\operatorname{Real}(\overrightarrow{\mathbf{A}})$. As shown by Y. Brudnyi and N. Krugliak [5], a space $A \in \operatorname{Real}(\overrightarrow{\mathbf{A}})$ if and only if it has a property of $K$-monotonicity: for any $x \in A, y \in \Sigma(\overrightarrow{\mathbf{A}})$ an inequality $K(1, t, y, \overrightarrow{\mathbf{A}}) \leq K(1, t, x, \overrightarrow{\mathbf{A}}), \forall t>0$, implies that $y \in A$ and $\|y\|_{A} \leq \lambda\|x\|_{A}$ with a constant $\lambda$ independent of $x, y$ (as usual, in the case $\lambda=1$ we add a word "exact" and in the case of $\lambda$ common for some set of spaces we say the $K$-monotonicity is uniform). Note that the initial spaces of couple $A_{0}, A_{1}$ are $K$-monotone if and only if their unit balls are closed in the space $\Sigma(\overrightarrow{\mathbf{A}})$; such a couple is said to be mutually closed.

Theorem 6. For any Banach couple $\operatorname{Real}(\overrightarrow{\mathbf{A}}) \subset \operatorname{Prim}(\overrightarrow{\mathbf{A}})$. For any mutually closed Banach couple $\operatorname{Real}(\overrightarrow{\mathbf{A}})=\operatorname{Prim}(\overrightarrow{\mathbf{A}})$.

The proof of this theorem is a mere union of the following two lemmas. 
Lemma 1. The sum and the intersection of any number of exact $K$-spaces also are $K$-spaces.

Proof. The assertion about intersection is obvious, while the assertion about sum can be proved by the use of a special result from [5] called $K$-divisibility. Namely, let all $A_{\omega}$ be $K$-spaces, $y \in \Sigma\left(A_{\omega}\right), x \in \Sigma(\overrightarrow{\mathbf{A}})$ and $K(1, t, x, \overrightarrow{\mathbf{A}}) \leq$ $K(1, t, y, \overrightarrow{\mathbf{A}})$. Due to definition of $\Sigma\left(A_{\omega}\right)$, for arbitrary $\varepsilon>0$, there exists a collection of $y_{\omega} \in A_{\omega}, \omega \in \Omega$, such that $y=\sum y_{\omega}$ and

$$
\|y\|_{\Sigma\left(A_{\omega}\right)} \geq(1-\varepsilon) \sum\left\|y_{\omega}\right\|_{A_{\omega}} .
$$

Since for any $t>0$ the $K$-functional is a norm in $\Sigma(\overrightarrow{\mathbf{A}})$, we obtain that

$$
K(1, t, x, \overrightarrow{\mathbf{A}}) \leq K(1, t, y, \overrightarrow{\mathbf{A}})=K\left(1, t, \sum y_{\omega}, \overrightarrow{\mathbf{A}}\right) \leq \sum K\left(1, t, y_{\omega}, \overrightarrow{\mathbf{A}}\right) .
$$

The property of $K$-divisibility gives us the existence of $x_{\omega}$ such that $x=$ $\sum x_{\omega}$ and $K\left(1, t, x_{\omega}, \overrightarrow{\mathbf{A}}\right) \leq \gamma K\left(1, t, y_{\omega}, \overrightarrow{\mathbf{A}}\right)$ for any $\omega$, where $\gamma$ is the so-called "constant of $K$-divisibility". Every $A_{\omega}$ is an exact $K$-space, so $\left\|x_{\omega}\right\|_{A_{\omega}} \leq$ $\left\|\gamma y_{\omega}\right\|_{A_{\omega}}$ and

$$
\|x\|_{\Sigma\left(A_{\omega}\right)} \leq \sum\left\|x_{\omega}\right\|_{A_{\omega}} \leq \gamma \sum\left\|y_{\omega}\right\|_{A_{\omega}} \leq \frac{\gamma}{1-\varepsilon}\|y\|_{\Sigma\left(A_{\omega}\right)} \cdot \cdot
$$

It is evident that, instead of exact $K$-monotonicity of spaces $A_{\omega}$, we could require only uniform one.

Lemma 2. For a space $A \in \pi(\overrightarrow{\mathbf{A}})$ to be $K$-monotone, it is necessary and sufficient that $A=\Sigma\left(\mathrm{M}_{\Phi_{\omega}}(\overrightarrow{\mathbf{A}})\right)$ over some function set $\left\{\Phi_{\omega}\right\} \subset \mathfrak{M}$.

Proof. The sufficiency follows from Lemma 1, because any Marcinkiewicz space is $K$-monotone. In order to prove the necessity let us define $\Phi_{\omega}(\alpha, \beta)=$ $1 / K\left(1 / \alpha, 1 / \beta, y_{\omega}, \overrightarrow{\mathbf{A}}\right)$ for each $y_{\omega}$ from the closed unit ball of $A$. Then for any $x$ from the unit ball of $\mathrm{M}_{\Phi_{\omega}}(\overrightarrow{\mathbf{A}})$, we have

$$
K(1, t, x, \overrightarrow{\mathbf{A}}) \leq 1 / \Phi_{\omega}(1,1 / t)=K\left(1, t, y_{\omega}, \overrightarrow{\mathbf{A}}\right)
$$

thus $\|x\|_{A} \leq\left\|y_{\omega}\right\|_{A} \leq 1$. This means that the unit balls of all $\mathrm{M}_{\Phi_{\omega}}(\overrightarrow{\mathbf{A}})$ are contained in the unit ball of $A$ which turns to be a union of them, and so $A$ is the "narrowest" space exactly containing all $\mathrm{M}_{\Phi_{\omega}}(\overrightarrow{\mathbf{A}})$, i.e. the sum of them.

\section{WEAK-TYPE INTERPOLATION}

In this section we discuss how an information about embedding functions of intermediate spaces can be used in the interpolation theory. In many cases just this information is most accessible and easy to compute. For 
example, in the couple $\left(L_{1}, L_{\infty}\right)$ one gets such information by study of an operator only on characteristic functions of measurable subsets. And even in the cases when a given space is well-known, we can encounter a situation this knowledge is useless for lack of appropriate interpolation theorems. So it makes sense to study linear operators acting from a space $A \in \pi(\overrightarrow{\mathbf{A}})$ to a space $B \in \pi(\overrightarrow{\mathbf{B}})$, when all we know is that $C_{A}(\alpha, \beta) \leq \Phi(\alpha, \beta), D_{B}(\alpha, \beta) \geq$ $\Psi(\alpha, \beta)$. Such operators will be said to be of weak type $(\Phi, \Psi)$; this is equivalent to their boundedness from $\Lambda_{\Phi}(\overrightarrow{\mathbf{A}})$ to $\mathrm{M}_{\Psi}(\overrightarrow{\mathbf{B}})$.

The weak-type interpolation relates to an operator being simultaneously of two weak types $\left(\Phi_{0}, \Psi_{0}\right)$ and $\left(\Phi_{1}, \Psi_{1}\right)$. Using general interpolation properties of Lorentz and Marcinkiewicz spaces [18], we get from this immediately that any such operator is also of weak type $(\Phi, \Psi)$, where

$$
\Phi(\alpha, \beta)=\Theta\left(\Phi_{0}(\alpha, \beta), \Phi_{1}(\alpha, \beta)\right), \quad \Psi(\alpha, \beta)=\Theta\left(\Psi_{0}(\alpha, \beta), \Psi_{1}(\alpha, \beta)\right)
$$

with arbitrary function $\Theta \in \mathfrak{M}$. However, for some particular classes of functions $\Theta$, we can establish stronger results. To this end we need to add some words about special properties of functions from $\mathfrak{M}$.

If $\Theta(\alpha, \beta) \in \mathfrak{M}$ then $\theta(t)=\Theta(1, t)$ is a quasiconcave function, that is it increases while $\theta(t) / t$ decreases. We say $\theta(t)$ (and $\Theta(\alpha, \beta)$ as well) is a quasi-power, if there exist positive numbers $\mu, \nu<1$ such that a function $\theta(t) t^{-\mu}$ is equivalent to some increasing function, and a function $\theta(t) t^{-\nu}$ is equivalent to some decreasing function. The numbers $p_{\theta}=\sup \mu, q_{\theta}=\inf \nu$ (over all possible numbers $\mu, \nu$ with aforesaid properties) are called lower and upper extension indices of the function $\theta(t)$. They can be found from the relations

$$
p_{\theta}=\lim _{t \rightarrow 0} \frac{\log m_{\theta}(t)}{\log t}, \quad q_{\theta}=\lim _{t \rightarrow \infty} \frac{\log m_{\theta}(t)}{\log t},
$$

where $m_{\theta}(t)=\sup _{s}(\theta(t s) / \theta(s))$. Obviously, $0<p_{\theta} \leq q_{\theta}<1$. Notice that via (8) such indices may be defined for any positive function, and then for arbitrary quasiconcave function, we get weaker inequalities $0 \leq p_{\theta} \leq q_{\theta} \leq 1$.

Theorem 7. Let a linear operator $T$ be of weak types $\left(\Phi_{0}, \Psi_{0}\right)$ and $\left(\Phi_{1}, \Psi_{1}\right)$ such that both the ratios $\Phi_{1}(t, 1) / \Phi_{0}(t, 1)$ and $\Psi_{1}(t, 1) / \Psi_{0}(t, 1)$ have positive extension indices. Let $\theta(t)=\Theta(1, t)$ be a quasi-power. Then $T: \Lambda_{\Phi}(\overrightarrow{\mathbf{A}}) \rightarrow$ $\Lambda_{\Psi}(\overrightarrow{\mathbf{B}})$ and $T: \mathrm{M}_{\Phi}(\overrightarrow{\mathbf{A}}) \rightarrow \mathrm{M}_{\Psi}(\overrightarrow{\mathbf{B}})$ with $\Phi, \Psi$ defined by $(\eta)$.

These assertions were proved via fundamental functions in [18] and via orbital methods (with some generalizations) in [10]. We would like only to emphasize once more the role of embedding functions taking as example the first assertion. Let $\vec{\Lambda}=\left(\Lambda_{\Phi_{0}}(\overrightarrow{\mathbf{A}}), \Lambda_{\Phi_{1}}(\overrightarrow{\mathbf{A}})\right)$ and define $G \in \pi(\vec{\Lambda})$ as a collection of $x \in \Lambda_{\Phi}(\overrightarrow{\mathbf{A}})$, for which $T x \in \Lambda_{\Psi}(\overrightarrow{\mathbf{B}})$ with the norm $\|x\|_{G}=$ $\max \left(\|x\|_{\Lambda_{\Phi}(\overrightarrow{\mathbf{A}})},\|T x\|_{\Lambda_{\Psi}(\overrightarrow{\mathbf{B}})}\right)$. Our goal is to estimate the embedding function $C_{G}(\alpha, \beta)$ in the couple $\vec{\Lambda}$. Using an inequality from [18]

$$
\|y\|_{\Lambda_{\Psi}(\overrightarrow{\mathbf{B}})} \leq C \Theta\left(\|y\|_{\mathrm{M}_{\Psi_{0}}(\overrightarrow{\mathbf{B}})},\|y\|_{\mathrm{M}_{\Psi_{1}}(\overrightarrow{\mathbf{B}})}\right)
$$


and the relation $\Lambda_{\Theta}(\vec{\Lambda})=\Lambda_{\Phi}(\overrightarrow{\mathbf{A}})$, we obtain that $\|x\|_{G} \leq C \Theta\left(\|x\|_{\Lambda_{\Phi_{0}}(\overrightarrow{\mathbf{A}})}\right.$, $\left.\|x\|_{\Lambda_{\Phi_{1}}(\overrightarrow{\mathbf{A}})}\right)$, whence $C_{G}(\alpha, \beta) \leq C \Theta(\alpha, \beta)$. This means that $G \supset \Lambda_{\Theta}(\vec{\Lambda})=$ $\Lambda_{\Phi}(\overrightarrow{\mathbf{A}})$, thus $\|T x\|_{\Lambda_{\Psi}(\overrightarrow{\mathbf{B}})} \leq C\|x\|_{\Lambda_{\Phi}(\overrightarrow{\mathbf{A}})}$ for all $x \in \Lambda_{\Phi}(\overrightarrow{\mathbf{A}})$ q.e.d.

In fact, Theorem 7 says that any operator $T$ satisfying its conditions is bounded from the couple $\left(\Lambda_{\Phi}(\overrightarrow{\mathbf{A}}), \mathrm{M}_{\Phi}(\overrightarrow{\mathbf{A}})\right)$ to the couple $\left(\Lambda_{\Psi}(\overrightarrow{\mathbf{B}}), \mathrm{M}_{\Psi}(\overrightarrow{\mathbf{B}})\right)$. Thus for any interpolation functor $\mathcal{F}$, we obtain that $T: A \rightarrow B$, where $A=$ $\mathcal{F}\left(\Lambda_{\Phi}(\overrightarrow{\mathbf{A}}), \mathrm{M}_{\Phi}(\overrightarrow{\mathbf{A}})\right), B=\mathcal{F}\left(\Lambda_{\Psi}(\overrightarrow{\mathbf{B}}), \mathrm{M}_{\Psi}(\overrightarrow{\mathbf{B}})\right)$. Now we give a description of all possible spaces $A, B$ obtained on this way.

As usual, a notation $\overrightarrow{\mathbf{A}}_{E}^{K}$ will be used for a space from $\operatorname{Real}(\overrightarrow{\mathbf{A}})$ with the norm

$$
\|x\|_{\overrightarrow{\mathbf{A}}_{E}^{K}}=\|K(1, t, x, \overrightarrow{\mathbf{A}})\|_{E} .
$$

For instance, $\mathrm{M}_{\Phi}(\overrightarrow{\mathbf{A}})=\overrightarrow{\mathbf{A}}_{L_{\infty}^{\varphi}}^{K}$, where $\varphi(t)=\Phi(1,1 / t)$. As shown in [18], the Lorentz space $\Lambda_{\Phi}(\overrightarrow{\mathbf{A}})$ can be represented in analogous form if the function $\Phi$ is a quasi-power: $\Lambda_{\Phi}(\overrightarrow{\mathbf{A}})=\overrightarrow{\mathbf{A}}_{L_{1}^{\varphi}}^{K}$, where the space $L_{1}$ is taken with respect to the measure $d t / t$. It is easy to check that, under conditions of Theorem 7 , both the functions $\Phi$ and $\Psi$ from (7) are quasi-powers, thus

$$
A=\mathcal{F}\left(\overrightarrow{\mathbf{A}}_{L_{1}^{\varphi}}^{K}, \overrightarrow{\mathbf{A}}_{L_{\infty}^{\varphi}}^{K}\right), \quad B=\mathcal{F}\left(\overrightarrow{\mathbf{B}}_{L_{1}^{\psi}}^{K}, \overrightarrow{\mathbf{B}}_{L_{\infty}^{\psi}}^{K}\right),
$$

and we may use the general reiteration theorem from [9] giving

$$
A=\overrightarrow{\mathbf{A}}_{E^{\varphi}}^{K}, \quad B=\overrightarrow{\mathbf{B}}_{E \psi}^{K}, \quad E=\mathcal{F}\left(L_{1}, L_{\infty}\right) .
$$

It remains only to add that, due to Calderón [6], a space $E$ has such a form if and only if it is rearrangement invariant (with respect to the measure $d t / t$ in our case).

The obtained representation of spaces $A, B$ is rather specific - it implies, in particular, that $A$ and $B$ are interpolation in their own couples $\left(\Lambda_{\Phi}(\overrightarrow{\mathbf{A}}), \mathrm{M}_{\Phi}(\overrightarrow{\mathbf{A}})\right)$ and $\left(\Lambda_{\Psi}(\overrightarrow{\mathbf{B}}), \mathrm{M}_{\Psi}(\overrightarrow{\mathbf{B}})\right)$ respectively. Returning to the initial problem of weak-type interpolation, we can enlarge the class of admissible spaces $A, B$ by use of operations of sum and intersection. Let $\Omega$ be a set of indices $\omega$ such that any $E_{\omega}$ is rearrangement invariant (in above sense) and any $\Theta_{\omega}$ is a quasi-power. Let $\varphi_{\omega}(t)=\Phi_{\omega}(1,1 / t), \psi_{\omega}(t)=\Psi_{\omega}(1,1 / t)$, where

$$
\Phi_{\omega}(\alpha, \beta)=\Theta_{\omega}\left(\Phi_{0}(\alpha, \beta), \Phi_{1}(\alpha, \beta)\right), \quad \Psi_{\omega}(\alpha, \beta)=\Theta_{\omega}\left(\Psi_{0}(\alpha, \beta), \Psi_{1}(\alpha, \beta)\right),
$$

and let $A_{\omega}=\overrightarrow{\mathbf{A}}_{E_{\omega}^{\psi_{\omega}}}^{K}, B_{\omega}=\overrightarrow{\mathbf{B}}_{E_{\omega}^{\psi_{\omega}}}^{K}$. As we know yet, $T: A_{\omega} \rightarrow B_{\omega}$ for each $\omega \in \Omega$. If all spaces $A_{\omega}$ are uniformly embedded in the space $\Sigma(\overrightarrow{\mathbf{A}})$, and all spaces $B_{\omega}$ are uniformly embedded in the space $\Sigma(\overrightarrow{\mathbf{B}})$, we may define

$$
A=\sum_{\omega \in \Omega} A_{\omega}, \quad B=\sum_{\omega \in \Omega} B_{\omega} .
$$


If at last, the interpolation constants of interpolation from $A_{\omega}$ to $B_{\omega}$ for all $\omega \in \Omega$ are bounded on the whole, we may apply Theorem 4 and thus obtain that $T: A \rightarrow B$. An analogous result is true if to replace all sums by intersections.

The above conditions on spaces $A_{\omega}, B_{\omega}$ are connected only with the properties of functions $\Theta_{\omega}$ telling on the corresponding constants in inequality (9). If to look through the proof of this inequality in [18], it is not difficult to see that the both kinds of uniformity are ensured by inequalities $\inf p_{\theta_{\omega}}>0, \sup q_{\theta_{\omega}}<1$ over all $\omega \in \Omega$ (for instance, when the set $\Omega$ is finite).

Notice that the spaces $A, B \in \operatorname{Real}(\overrightarrow{\mathbf{A}})$ due to Lemma 1. By use of the $K$-divisibility one can easy prove that in the case of sum

$$
A=\overrightarrow{\mathbf{A}}_{E}^{K}, \quad B=\overrightarrow{\mathbf{B}}_{F}^{K}, \quad E=\sum_{\omega \in \Omega} E_{\omega}^{\varphi_{\omega}}, \quad F=\sum_{\omega \in \Omega} E_{\omega}^{\psi_{\omega}},
$$

while in the case of intersection

$$
E=\Delta_{\omega \in \Omega} E_{\omega}^{\varphi_{\omega}}, \quad F=\Delta_{\omega \in \Omega} E_{\omega}^{\psi_{\omega}} .
$$

We see that in the both cases the structure of spaces $E, F$ is rather complicated and may be not easy to verify. Therefore we will give another assertion not requiring any information concerning this structure.

Theorem 8. Under conditions of Theorem 7 let $\varphi(t)=\Phi(1,1 / t), \psi(t)=$ $\Psi(1,1 / t)$ and let the triple $\left(L_{1}^{\varphi}, L_{\infty}^{\varphi}, E\right)$ be interpolation with respect to the triple $\left(L_{1}^{\psi}, L_{\infty}^{\psi}, F\right)$. Then $T: \overrightarrow{\mathbf{A}}_{E}^{K} \rightarrow \overrightarrow{\mathbf{B}}_{F}^{K}$.

Proof. Due to the general principle of N. Aronszajn and E. Gagliardo [1], there exists an interpolation functor $\mathcal{F}$ such that $E \subset \mathcal{F}\left(L_{1}^{\varphi}, L_{\infty}^{\varphi}\right)$, $F \supset \mathcal{F}\left(L_{1}^{\psi}, L_{\infty}^{\psi}\right)$, thus $\overrightarrow{\mathbf{A}}_{E}^{K} \subset A, \overrightarrow{\mathbf{B}}_{F}^{K} \supset B$, where the spaces $A, B$ are defined by (10) with this functor. Our assertion follows now from the boundedness of $T$ from $A$ to $B$ which was proved above.

The required interpolation property of the triple $\left(L_{1}^{\varphi}, L_{\infty}^{\varphi}, E\right)$ with respect to the triple $\left(L_{1}^{\psi}, L_{\infty}^{\psi}, F\right)$ may be obtained, for instance, from Theorem 3 by comparison of the embedding functions of spaces $E, F$. Let $D_{E}(\alpha, \beta)$ be calculated in the couple $\left(L_{1}^{\varphi}, L_{\infty}^{\varphi}\right)$ and let $C_{F}(\alpha, \beta)$ be calculated in the couple $\left(L_{1}^{\psi}, L_{\infty}^{\psi}\right)$. If

$$
\int_{0}^{\infty} \frac{C_{F}(\alpha, \beta)}{D_{E}(\alpha, \beta)} \frac{d \beta}{\beta}=\int_{0}^{\infty} \frac{C_{F}(\alpha, \beta)}{D_{E}(\alpha, \beta)} \frac{d \alpha}{\alpha}<\infty,
$$

then $E \subset \Lambda_{C_{F}}\left(L_{1}^{\varphi}, L_{\infty}^{\varphi}\right)$. Any linear operator $T$ which is bounded from the couple $\left(L_{1}^{\varphi}, L_{\infty}^{\varphi}\right)$ to the couple $\left(L_{1}^{\psi}, L_{\infty}^{\psi}\right)$ acts from $\Lambda_{C_{F}}\left(L_{1}^{\varphi}, L_{\infty}^{\varphi}\right)$ to $\Lambda_{C_{F}}\left(L_{1}^{\psi}, L_{\infty}^{\psi}\right) \subset F$, hence $T: E \rightarrow F$. We refer the reader to [20], where this method was realized for some Orlicz spaces. 


\section{Generalization of the KREIN-SEMENOV INTERPOLATION THEOREM}

All results of the preceding section are based on Theorem 7 which requirements on the functions $\Phi_{i}, \Psi_{i}(i=0,1)$ as well as the definition (7) of the functions $\Phi, \Psi$ are absolutely symmetrical. For the case of rearrangement invariant spaces in the couple $\overrightarrow{\mathbf{A}}=\overrightarrow{\mathbf{B}}=\left(L_{1}, L_{\infty}\right)$, S. G. Krein and E. M. Semenov ([13], see also [12]) have established another kind of weak-type interpolation, where the basic conditions concerned only the initial space $A$, while the resulting space $B$ was defined via $A$ by a special construction. This result was later partly generalized in [8], and then the spaces constructed by S. G. Krein and E. M. Semenov were used in [13] as parameters of the $k$-method of interpolation proposed by C. Bennett [3]. In this section we give a generalization of the Krein-Semenov method to arbitrary $K$-spaces (by the way we remove the condition on the function $\Phi_{1}(t, 1) / \Phi_{0}(t, 1)$ to be increasing that was present in all above mentioned results).

Let $\mathfrak{N}$ be the set of all functions $f(t): \mathbb{R}^{+} \rightarrow \mathbb{R}^{+}$, for which $t f(t)$ is a quasiconcave function. For each Banach function space $E$ with nonempty intersection $E \cap \mathfrak{N}$, define a function

$$
\sigma_{E}(s)=\sup _{f \in E \cap \mathfrak{N}} \frac{\|f(t / s)\|_{E}}{\|f(t)\|_{E}}, \quad s>0,
$$

which obviously is quasiconcave too. Its extension indices may be regarded as some global characteristics of the space $E$ and will be denoted by $p_{E}$ (lower) and $q_{E}$ (upper). One can show that for the case of rearrangement invariant space $E$, these characteristics coincide with the Boyd indices [4] of $E$, provided the latter are less than 1 .

Theorem 9. Let a linear operator $T$ be of weak types $\left(\Phi_{0}, \Psi_{0}\right)$ and $\left(\Phi_{1}, \Psi_{1}\right)$ such that $p_{\Phi_{0}(t, 1)}>q_{\Phi_{1}(t, 1)}$. Assume there exists a positive function $\delta(t)$ such that

$$
\Psi_{1}(\delta(t), 1) / \Psi_{0}(\delta(t), 1)=\Phi_{1}(t, 1) / \Phi_{0}(t, 1)
$$

for all $t>0$. If a Banach function space $E$ has the characteristics $p_{E}>$ $q_{\Phi_{1}(t, 1)}, q_{E}<p_{\Phi_{0}(t, 1)}$, then $T: A \rightarrow B$, where $A \in \pi(\overrightarrow{\mathbf{A}}), B \in \pi(\overrightarrow{\mathbf{B}})$ with the norms

$$
\|x\|_{A}=\left\|\frac{1}{t} K(1, t, x, \overrightarrow{\mathbf{A}})\right\|_{E}, \quad\|x\|_{B}=\left\|\frac{\kappa(t)}{t} K(1, \delta(t), x, \overrightarrow{\mathbf{B}})\right\|_{E}
$$

and

$$
\kappa(t)=\frac{\Psi_{0}(1,1 / \delta(t))}{\Phi_{0}(1,1 / t)}=\frac{\Psi_{1}(1,1 / \delta(t))}{\Phi_{1}(1,1 / t)} .
$$

Proof. On the first stage let us prove the theorem for the case when the functions $\alpha_{0}(t)=\Phi_{0}(t, 1)$ and $\alpha_{1}(t)=\Phi_{1}(t, 1)$ are quasi-powers, i.e. $p_{\alpha_{1}}>$ 
$0, q_{\alpha_{0}}<1$. Take some $x \in A$ and for arbitrary fixed $t>0$ define two functions

$$
a(s)=\left\{\begin{array}{ll}
K(1, s, x, \overrightarrow{\mathbf{A}}) & \text { if } s \leq t \\
K(1, t, x, \overrightarrow{\mathbf{A}}) & \text { if } s \geq t,
\end{array} \quad b(s)= \begin{cases}s K(1, t, x, \overrightarrow{\mathbf{A}}) / t & \text { if } s \leq t \\
K(1, s, x, \overrightarrow{\mathbf{A}}) & \text { if } s \geq t\end{cases}\right.
$$

Both the functions $a(s), b(s)$ are increasing and concave and $K(1, s, x, \overrightarrow{\mathbf{A}}) \leq$ $a(s)+b(s)$. Thus in virtue of $K$-divisibility, there exists a decomposition $x=x_{0}+x_{1} ; x_{0}, x_{1} \in \Sigma(\overrightarrow{\mathbf{A}})$ such that

$$
K\left(1, s, x_{0}, \overrightarrow{\mathbf{A}}\right) \leq \gamma a(s), \quad K\left(1, s, x_{1}, \overrightarrow{\mathbf{A}}\right) \leq \gamma b(s)
$$

Show that $x_{0} \in \Lambda_{\Phi_{0}}(\overrightarrow{\mathbf{A}}), x_{1} \in \Lambda_{\Phi_{1}}(\overrightarrow{\mathbf{A}})$.

From the definition of $A$, we have $K(1, s, x, \overrightarrow{\mathbf{A}}) \in E^{1 / s}$, thus $a(s) \in E_{0}=$ $E^{1 / s} \cap L_{\infty}, b(s) \in E_{1}=E^{1 / s} \cap L_{\infty}^{1 / s}$. Hence $x_{0} \in A^{0}=\overrightarrow{\mathbf{A}}_{E_{0}}^{K}, x_{1} \in A^{1}=\overrightarrow{\mathbf{A}}_{E_{1}}^{K}$ and it suffices to prove that $A^{i} \subset \Lambda_{\Phi_{i}}(\overrightarrow{\mathbf{A}}), i=0,1$. We shall do this by the use of Theorem 3. To this end we estimate

$$
\begin{aligned}
D_{A^{0}}(\tau, 1)=\inf _{x \in A^{0}} \frac{\|K(1, s, x, \overrightarrow{\mathbf{A}})\|_{E_{0}}}{K(1, \tau, x, \overrightarrow{\mathbf{A}}) / \tau} & \geq \max \left\{\tau, \inf _{x \in A}\left\|\frac{K(1, s, x, \overrightarrow{\mathbf{A}}) / s}{K(1, \tau, x, \overrightarrow{\mathbf{A}}) / \tau}\right\|_{E}\right\} \\
& \geq \max \left\{\tau,\|\min (\tau / s, 1)\|_{E}\right\}
\end{aligned}
$$

(the last inequality is due to concavity of $K(1, s, x, \overrightarrow{\mathbf{A}})$ as a function of $s$ ). The function $\min (1 / s, 1) \in \mathfrak{N}$, therefore

$$
\|\min (\tau / s, 1)\|_{E} \geq \frac{\|\min (1 / s, 1)\|_{E}}{\sigma_{E}(1 / \tau)} .
$$

Recall that in our case the space $E^{1 / s}$ is a parameter of the real interpolation, thus the value $\|\min (1 / s, 1)\|_{E}$ is well-defined. Now we can estimate the integral

$$
\begin{aligned}
\int_{0}^{\infty} \frac{\Phi_{0}(\alpha, \beta)}{D_{A^{0}}(\alpha, \beta)} \frac{d \alpha}{\alpha} & =\int_{0}^{\infty} \frac{\Phi_{0}(\tau, 1)}{D_{A^{0}}(\tau, 1)} \frac{d \tau}{\tau} \\
& \leq C \int_{0}^{\infty} \alpha_{0}(\tau) \min \left(1 / \tau, \sigma_{E}(1 / \tau)\right) d \tau / \tau \\
& =C \int_{0}^{1} \alpha_{0}(\tau) \sigma_{E}(1 / \tau) d \tau / \tau+C \int_{1}^{\infty} \alpha_{0}(\tau) d \tau / \tau^{2} \\
& <\infty
\end{aligned}
$$

since $p_{\alpha_{0}}>q_{E}, q_{\alpha_{0}}<1$. Thus Theorem 3 gives us an embedding $A^{0} \subset$ $\Lambda_{\Phi_{0}}(\overrightarrow{\mathbf{A}})$. 
Analogously,

$$
D_{A^{1}}(\tau, 1)=\inf _{x \in A^{1}} \frac{\|K(1, s, x, \overrightarrow{\mathbf{A}})\|_{E_{1}}}{K(1, \tau, x, \overrightarrow{\mathbf{A}}) / \tau} \geq \max \left\{1,\|\min (\tau / s, 1)\|_{E}\right\},
$$

whence

$$
\begin{aligned}
\int_{0}^{\infty} \frac{\Phi_{1}(\alpha, \beta)}{D_{A^{1}}(\alpha, \beta)} \frac{d \alpha}{\alpha} & \leq C \int_{0}^{\infty} \alpha_{1}(\tau) \min \left(1, \sigma_{E}(1 / \tau)\right) d \tau / \tau \\
& =C \int_{0}^{1} \alpha_{1}(\tau) d \tau / \tau+C \int_{1}^{\infty} \alpha_{1}(\tau) \sigma_{E}(1 / \tau) d \tau / \tau \\
& <\infty
\end{aligned}
$$

since $p_{\alpha_{1}}>0, q_{\alpha_{1}}<p_{E}$. We have proved the second embedding $A^{1} \subset$ $\Lambda_{\Phi_{1}}(\overrightarrow{\mathbf{A}})$ as well.

So the operator $T$ is defined on $x_{0}, x_{1}$, and the following inequalities hold:

$$
\left\|T x_{i}\right\|_{\mathrm{M}_{\Psi_{i}}(\overrightarrow{\mathbf{B}})} \leq C\left\|x_{i}\right\|_{\Lambda_{\Phi_{i}}(\overrightarrow{\mathbf{A}})}, \quad i=0,1 .
$$

Keeping the previously fixed value of $t$, we are now able to estimate

$$
\begin{aligned}
\frac{\kappa(t)}{t} K(1, \delta(t), T x, \overrightarrow{\mathbf{B}}) & \leq \frac{\kappa(t)}{t}\left[K\left(1, \delta(t), T x_{0}, \overrightarrow{\mathbf{B}}\right)+K\left(1, \delta(t), T x_{1}, \overrightarrow{\mathbf{B}}\right)\right] \\
& \leq \frac{\kappa(t)}{t}\left[\frac{\left\|T x_{0}\right\|_{\mathrm{M}_{\Psi_{0}}(\overrightarrow{\mathbf{B}})}}{\Psi_{0}(1,1 / \delta(t))}+\frac{\left\|T x_{1}\right\|_{\mathrm{M}_{\Psi_{1}}(\overrightarrow{\mathbf{B}})}}{\Psi_{1}(1,1 / \delta(t))}\right] \\
& \leq C\left[\frac{1}{\alpha_{0}(t)}\left\|x_{0}\right\|_{\Lambda_{\Phi_{0}}(\overrightarrow{\mathbf{A}})}+\frac{1}{\alpha_{1}(t)}\left\|x_{1}\right\|_{\Lambda_{\Phi_{1}}(\overrightarrow{\mathbf{A}})}\right] .
\end{aligned}
$$

Since both the functions $\Phi_{0}, \Phi_{1}$ are quasi-powers, we have that $\Lambda_{\Phi_{i}}(\overrightarrow{\mathbf{A}})=$ $\overrightarrow{\mathbf{A}}_{L_{1}^{\varphi_{i}}}^{K}, i=0,1$, where $\varphi_{i}(s)=\Phi_{i}(1,1 / s)=\alpha_{i}(s) / s$. Therefore

$$
\begin{aligned}
& \left\|x_{0}\right\|_{\Lambda_{\Phi_{0}}(\overrightarrow{\mathbf{A}})} \leq C \int_{0}^{\infty} K\left(1, s, x_{0}, \overrightarrow{\mathbf{A}}\right) \alpha_{0}(s) d s / s^{2} \leq \gamma C \int_{0}^{\infty} a(s) \alpha_{0}(s) d s / s^{2}, \\
& \left\|x_{1}\right\|_{\Lambda_{\Phi_{1}}(\overrightarrow{\mathbf{A}})} \leq C \int_{0}^{\infty} K\left(1, s, x_{1}, \overrightarrow{\mathbf{A}}\right) \alpha_{1}(s) d s / s^{2} \leq \gamma C \int_{0}^{\infty} b(s) \alpha_{1}(s) d s / s^{2},
\end{aligned}
$$

which allows to obtain

$$
\begin{aligned}
& \frac{\kappa(t)}{t} K(1, \delta(t), T x, \overrightarrow{\mathbf{B}}) \\
& \leq C\left[\int_{0}^{\infty} a(s) m_{\alpha_{0}}(s / t) d s / s^{2}+\int_{0}^{\infty} b(s) m_{\alpha_{1}}(s / t) d s / s^{2}\right] \\
& =C\left[\int_{0}^{1} \frac{1}{t s} K(1, t s, x, \overrightarrow{\mathbf{A}}) m_{\alpha_{0}}(s) d s / s+\int_{1}^{\infty} \frac{1}{t} K(1, t, x, \overrightarrow{\mathbf{A}}) m_{\alpha_{0}}(s) d s / s^{2}\right. \\
& \left.\quad+\int_{0}^{1} \frac{1}{t} K(1, t, x, \overrightarrow{\mathbf{A}}) m_{\alpha_{1}}(s) d s / s+\int_{1}^{\infty} \frac{1}{t s} K(1, t s, x, \overrightarrow{\mathbf{A}}) m_{\alpha_{1}}(s) d s / s\right] .
\end{aligned}
$$


Now we have everything to estimate $\|T x\|_{B}$ taking in account that $K(1, t$, $x, \overrightarrow{\mathbf{A}}) / t \in \mathfrak{N}:$

$$
\begin{aligned}
\|T x\|_{B}= & \left\|\frac{\kappa(t)}{t} K(1, \delta(t), T x, \overrightarrow{\mathbf{B}})\right\|_{E} \\
\leq & C\left\|\frac{1}{t} K(1, t, x, \overrightarrow{\mathbf{A}})\right\|_{E}\left[\int_{0}^{1} \sigma_{E}\left(\frac{1}{s}\right) m_{\alpha_{0}}(s) \frac{d s}{s}+\int_{1}^{\infty} m_{\alpha_{0}}(s) \frac{d s}{s^{2}}\right. \\
& \left.+\int_{0}^{1} m_{\alpha_{1}}(s) \frac{d s}{s}+\int_{1}^{\infty} \sigma_{E}\left(\frac{1}{s}\right) m_{\alpha_{1}}(s) \frac{d s}{s}\right] .
\end{aligned}
$$

In order to finish the first stage of the proof, we only have to ascertain that all the four integrals in the brackets are finite. But this is ensured by the given inequalities for indices.

Let us pass to the second stage of the proof to remove the extra conditions $p_{\alpha_{1}}>0, q_{\alpha_{0}}<1$. Put $\lambda=\frac{1}{2} \min \left(p_{\alpha_{0}}-q_{E}, p_{E}-q_{\alpha_{1}}\right)$ and show that all conditions of our theorem are fulfilled, if to replace $\Phi_{0}, \Phi_{1}, \Psi_{0}, \Psi_{1}$ by

$$
\tilde{\Phi}_{0}=\Phi_{0}^{1-\lambda} \Phi_{1}^{\lambda}, \quad \tilde{\Phi}_{1}=\Phi_{0}^{\lambda} \Phi_{1}^{1-\lambda}, \quad \tilde{\Psi}_{0}=\Psi_{0}^{1-\lambda} \Psi_{1}^{\lambda}, \quad \tilde{\Psi}_{1}=\Psi_{0}^{\lambda} \Psi_{1}^{1-\lambda} .
$$

These equalities may be considered as partial cases of the relation (7), thus the operator $T$ remains to be of weak types $\left(\tilde{\Phi}_{0}, \tilde{\Psi}_{0}\right)$ and $\left(\tilde{\Phi}_{1}, \tilde{\Psi}_{1}\right)$. It is possible to take a function $\delta(t)$ as before, since

$$
\frac{\tilde{\Psi}_{1}(\delta(t), 1)}{\tilde{\Psi}_{0}(\delta(t), 1)}=\left[\frac{\Psi_{1}(\delta(t), 1)}{\Psi_{0}(\delta(t), 1)}\right]^{1-2 \lambda}=\left[\frac{\Phi_{1}(t, 1)}{\Phi_{0}(t, 1)}\right]^{1-2 \lambda}=\frac{\tilde{\Phi}_{1}(t, 1)}{\tilde{\Phi}_{0}(t, 1)} .
$$

Just so easy one can ascertain that the second function $\kappa(t)$ does not change as well. It only remains to find out new relations between indices.

Denote like before $\tilde{\Phi}_{i}(t, 1)=\tilde{\alpha}_{i}(t), i=0,1$. By a direct calculation we obtain that

$$
\begin{array}{ll}
p_{\tilde{\alpha}_{0}}=(1-\lambda) p_{\alpha_{0}}+\lambda p_{\alpha_{1}}, & q_{\tilde{\alpha}_{0}}=(1-\lambda) q_{\alpha_{0}}+\lambda q_{\alpha_{1}}, \\
p_{\tilde{\alpha}_{1}}=\lambda p_{\alpha_{0}}+(1-\lambda) p_{\alpha_{1}}, & q_{\tilde{\alpha}_{1}}=\lambda q_{\alpha_{0}}+(1-\lambda) q_{\alpha_{1}},
\end{array}
$$

whence

$$
p_{\tilde{\alpha}_{0}}-q_{E} \geq p_{\alpha_{0}}-\lambda-q_{E} \geq \lambda, \quad p_{E}-q_{\tilde{\alpha}_{1}} \geq p_{E}-\lambda-q_{\alpha_{1}} \geq \lambda,
$$

and so $p_{\tilde{\alpha}_{0}}>q_{E}, q_{\tilde{\alpha}_{1}}<p_{E}$. On the other hand $p_{\tilde{\alpha}_{1}} \geq \lambda p_{\alpha_{0}}>0$ even if $p_{\alpha_{1}}=0$, and $q_{\tilde{\alpha}_{0}} \leq 1-\lambda+\lambda q_{\alpha_{1}}<1$ even if $q_{\alpha_{0}}=1$. Thus we can carry out the first stage of the proof for the functions $\tilde{\Phi}_{i}, \tilde{\Psi}_{i}, i=0,1$ and get the desired result.

Remark. As all over the real interpolation, any result of the last two sections remains to be true when $T$ is a bounded nonlinear operator in the sense of Gagliardo-Peetre (see [5]). 


\section{REFERENCES}

1. N. Aronszajn and E. Gagliardo, Interpolation spaces and interpolation methods, Ann. Mat. Pura Appl. (4) 68 (1965), 51-117.

2. S. V. Astashkin and V. I. Ovchinnikov, Functorial approach to interpolation of operators of weak type, Sibirsk. Mat. Zh. 32 (1991), 12-23 (Russian); English transl. Siberian Math. J. 32 (1991), 360-370.

3. C. Bennett, Banach function spaces and interpolation methods. I. The abstract theory, J. Funct. Anal. 17 (1974), 409-440.

4. D. W. Boyd, Indices of function spaces and their relationship to interpolation, Canad. J. Math. 21 (1969), 1245-1254.

5. Y. A. Brudnyi and N. Y. Krugliak, Interpolation Functors and Interpolation Spaces, I, North-Holland Math. Library, \#47, North-Holland, Amsterdam, 1991.

6. A. P. Calderón, Spaces between $L^{1}$ and $L^{\infty}$ and the theorems of Marcinkiewicz, Studia Math. 26 (1966), 273-299.

7. A. A. Dmitriev, The interpolation of one-dimensional operators, Voronež. Gos. Univ. Trudy Naučn.-Issled. Inst. Mat. VGU Vyp. 11 Sb. Statei Funkcional. Anal. i Prilozen 11 (1973), 31-43. (Russian)

8. V. I. Dmitriev and S. G. Krein, Interpolation of operators of weak type, Anal. Math. 4 (1978), 83-99.

9. V. I. Dmitriev and V. I. Ovchinnikov, On interpolation in spaces of the real method, Dokl. Akad. Nauk SSSR 246 (1979), 794-797 (Russian); English transl., Soviet. Math. Dokl. 20 (1979), 538-542.

10. S. Janson, Minimal and maximal methods of interpolation, J. Funct. Anal. 44 (1981), 50-73.

11. S. G. Krein, On a concept of a normal scale of spaces, Dokl. Akad. Nauk SSSR 132 (1960), 510-513 (Russian); English transl., Soviet. Math. Dokl. 1 (1960), 586-589.

12. S. G. Krein, Y. I. Petunin and E. M. Semenov, Interpolation of Linear Operators, Nauka, Moscow, 1978 (Russian); English transl., Amer. Math. Soc., Providence, 1982.

13. S. G. Krein and E. M. Semenov, Interpolation of operators of weakened type, Funkcional. Anal. i Priložen 7 (1973), 89-90 (Russian); English transl., Funct. Anal. Appl. 7 (1973), 161-162.

14. L. Maligranda, A Bibliography on "Interpolation of Operators and Applications (1926-1988)", LuleåUniv., Sweden, 1988.

15. V. I. Ovchinnikov, Interpolation theorems resulting from Grothendieck's inequality, Funkcional. Anal. i Prilozhen. 10 (1976), 45-54 (Russian); English transl., Funct. Anal. Appl. 10 (1976), 287-294.

16. J. Peetre, Sur le nombre de paramétres dans la définition de certains espaces d'interpolation, Ricerche Mat. 12 (1963), 248-261.

17. E. Pustylnik, Interpolation theorems in massives of Banach spaces, Dokl. Akad. Nauk SSSR 224 (1975), 1024-1027 (Russian); English transl., Soviet. Math. Dokl. 16 (1975), 1359-1363.

18. E. Pustylnik, Minimal and maximal intermediate Banach spaces, Ukrain. Mat. Zh. 29 (1977), 129-137 (Russian); English transl., Ukrainian Math. J. 29 (1977), 102107.

19. E. Pustylnik, Estimation of the position of intermediate spaces for a Banach couple, Studia Math. 107 (1993), 137-155.

20. E. Pustylnik, Functions of a second order elliptic operator in rearrangement invariant spaces, Integral equations Operator Theory 22 (1995), 476-498.

21. E. M. Semenov, Interpolation of linear operators in symmetric spaces, Dokl. Akad. Nauk SSSR 164 (1965), 746-749 (Russian); English transl., Soviet. Math. Dokl. 6 (1965), 1294-1297. 
Department of Mathematics

Technion, IsRael Institute of Technology

32000 HAIFA, ISRAEL

E-mail address: evg@techunix.technion.ac.il 


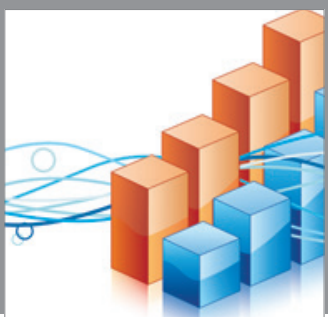

Advances in

Operations Research

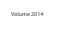

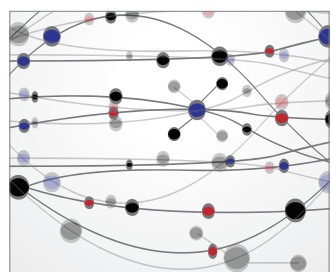

\section{The Scientific} World Journal
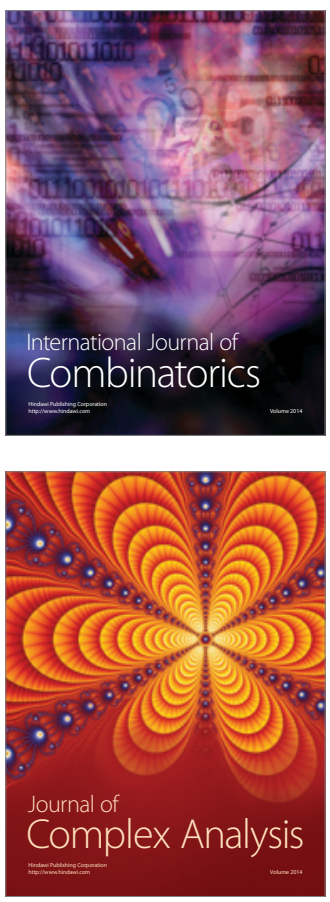

International Journal of

Mathematics and

Mathematical

Sciences
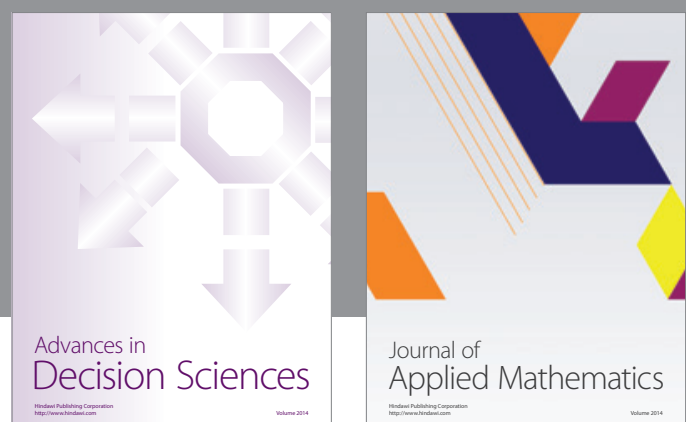

Journal of

Applied Mathematics
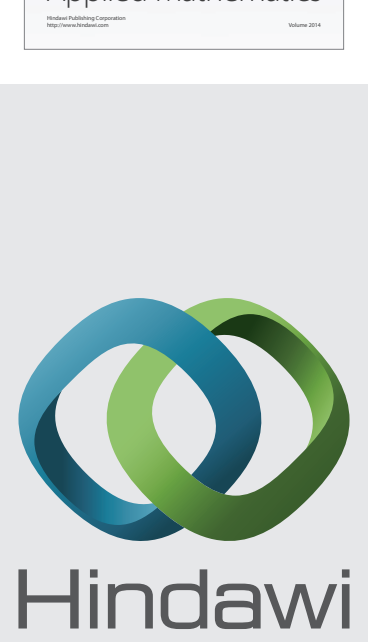

Submit your manuscripts at http://www.hindawi.com
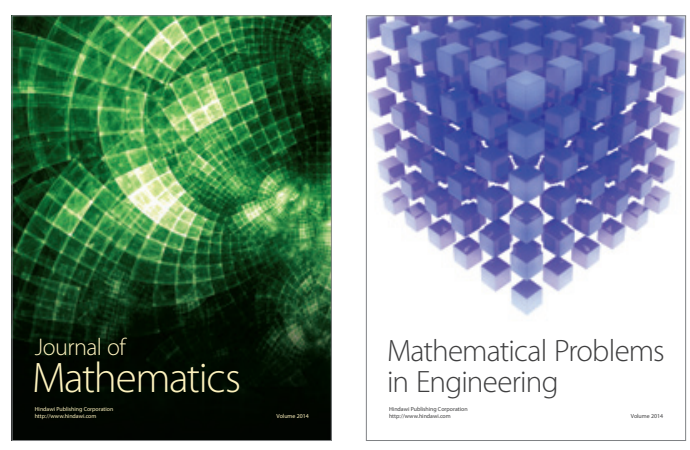

Mathematical Problems in Engineering
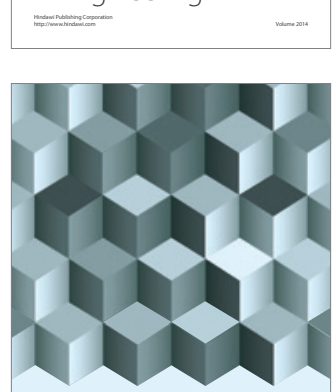

Journal of

Function Spaces
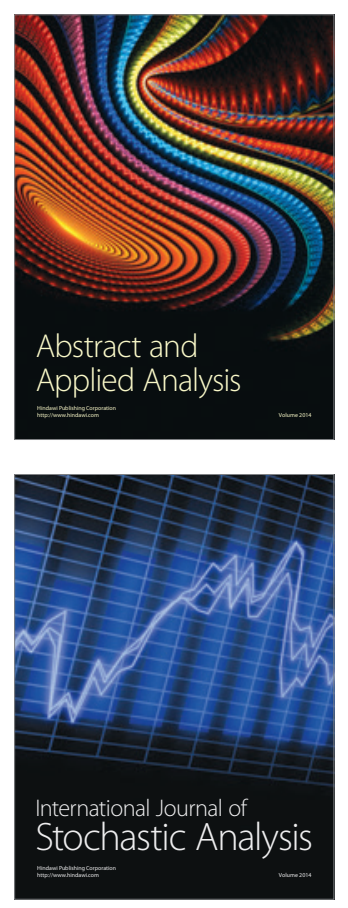

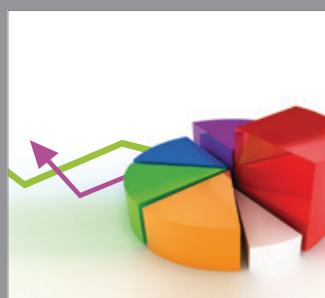

ournal of

Probability and Statistics

Promensencen
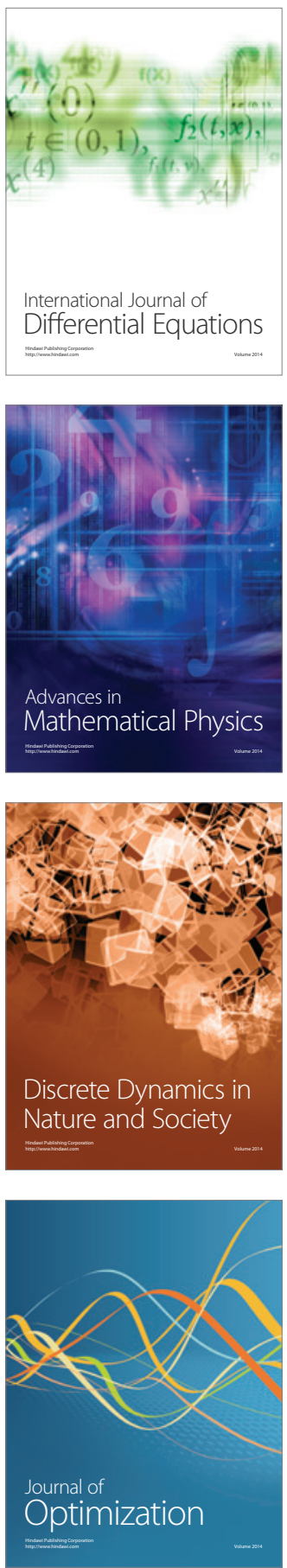